\title{
Les pièges de l'instrumentalisme... et de la répression : note sur une crise indienne : l'affaire d'Ayodhya (décembre 1992 - janvier 1993)
}

\section{Christophe Jaffrelot}

\section{(2) OpenEdition}

Journals

Édition électronique

URL : http://journals.openedition.org/conflits/519

DOI : 10.4000/conflits.519

ISSN : 1777-5345

Éditeur :

CCLS - Centre d'études sur les conflits lilberté et sécurité, L'Harmattan

Édition imprimée

Date de publication : 6 décembre 1992

ISSN : 1157-996X

Référence électronique

Christophe Jaffrelot, « Les pièges de l'instrumentalisme... et de la répression : note sur une crise indienne : l'affaire d'Ayodhya (décembre 1992 - janvier 1993)», Cultures \& Conflits [En ligne], 08 | hiver 1992, mis en ligne le 07 janvier 2003, consulté le 30 mars 2021. URL : http://journals.openedition.org/ conflits/519; DOI : https://doi.org/10.4000/conflits.519

Ce document a été généré automatiquement le 30 mars 2021.

Creative Commons License 


\title{
Les pièges de l'instrumentalisme... et de la répression : note sur une crise indienne : l'affaire d'Ayodhya (décembre 1992 - janvier 1993)
}

\author{
Christophe Jaffrelot
}

1 La scène publique de l'Inde est dominée depuis la fin des années 1980 par un conflit entre hindous et musulmans dont l'objet est le site d'Ayodhya ${ }^{1}$. Dans cette petite ville d'Uttar Pradesh, une mosquée, la Babri Masjid, du nom de Babur, le premier empereur moghol qui l'a faite construire, a été édifiée au XVIe siècle sur un lieu qui aurait été celui de la naissance du dieu Ram (litt. Ramjanmabhoomi). Cette croyance, très populaire dans l'Inde du nord où Ram fait l'objet d'une vénération particulière, fut habilement renforcée en 1949 lorsque des nationalistes hindous installèrent dans la mosquée des idoles du dieu et de sa parèdre, Sita. L'événement fut perçu comme une apparition divine du côté hindou et comme un blasphème par les musulmans. Ce lieu saint fut aussitôt fermé au culte au moyen de scellés, mais, dans les années $1980^{2}$, les nationalistes hindous recommencèrent à arguer de cette "apparition" et de la présence de piliers issus d'un temple hindou qui aurait été détruit par les musulmans ${ }^{3}$

2 , pour revendiquer ce site en vue d'y édifier un grand temple.

3 Les mouvements nationalistes hindous en question appartiennent à la nébuleuse du Rashtriya Swayamsevak Sangh (RSS - Association des volontaires nationaux). Cette organisation, fondée en 1925, s'est développée sous la forme d'un réseau de branches locales où les cadres du RSS (appelés pracharak, prêcheurs) dispensent quotidiennement à de jeunes hindous une discipline physique et idéologique dont les méfaits des envahisseurs musulmans et la gloire passée des hindous sont parmi les principaux sujets. Ce mouvement s'est doté au fil du temps de nombreuses filiales dont les plus importantes sont aujourd'hui le Bharatiya Janata Party (BJP - Parti du peuple indien) et la Vishva Hindu Parishad (VHP - Association hindoue universelle). Le premier constitue un front politique appelé à représenter le RSS dans la compétition électorale, 
tandis que la seconde a vocation à regrouper les représentants des sectes hindoues. Ce réseau religieux, qui s'est beaucoup étoffé au cours des années 1980, a joué un rôle crucial dans les campagnes nationalistes hindoues autour du site d'Ayodhya.

Celles-ci s'inscrivent dans le cadre d'une véritable "stratégie instrumentaliste". Les organisations nationalistes hindoues s'emploient en effet à mobiliser la communauté majoritaire en manipulant des symboles d'identité au premier rang desquels figure Ram, qui fut par exemple représenté, tel un prisonnier de la mosquée, derrière des barreaux. De façon significative, cette instrumentalisation revêtit un caractère particulièrement élaboré et militant dans le cadre des dernières campagnes électorales. En janvier 1989, la VHP annonça qu'elle poserait la première pierre du temple conçu par ses architectes, le 9 novembre de la même année, soit 15 jours avant les élections ; au cours de l'été et de l'automne, les religieux ralliés par cette organisation parcoururent l'Inde pour présider à des petites cérémonies au cours desquelles des briques, marquées du nom de Ram, étaient dites consacrées. Des milliers de personnes participèrent à ces Ram Shila Puja. Les sollicitations de dons religieux dont elles étaient assorties reçurent une réponse massive. Dans ce contexte, le BJP, dont les candidats étaient souvent associés à la mise en oeuvre de ces Ram Shila Puja, recueillit un nombre de voix sans précédent (11 \% des suffrages exprimés). En 1990, cette mobilisation populaire fut le principal facteur du succès de ce parti lors des élections dans trois Etats fédérés d'Inde du nord (l'Himachal Pradesh, le Madhya Pradesh et le Rajasthan). Cette variable opéra encore en 1991, portant le BJP à $22 \%$ des voix, soit 119 sièges à la Chambre basse où un de ses chefs, Advani, devenait le leader de l'opposition. Simultanément, le parti prenait le contrôle d'un quatrième Etat fédéré, le plus peuplé de l'Inde, l'Uttar Pradesh, où se trouve précisément Ayodhya.

Ces succès électoraux, en bonne part liés à la "stratégie instrumentaliste" déployée par la nébuleuse du RSS, portaient en germe une contradiction : ils impliquaient que le BJP se transformât en parti de gouvernement dans les Etats qu'il contrôlait, tout en poursuivant ses efforts de mobilisation autour de l'enjeu d'Ayodhya pour garder l'allégeance de ses militants et des religieux et, au-delà, pour envisager de conquérir le pouvoir central. La crise de décembre 1992, en exacerbant ce paradoxe, a révélé les limites de la stratégie instrumentaliste des nationalistes hindous avant que la répression orchestrée par New Delhi ne les transforme en victimes.

Les pièges de la stratégie instrumentaliste. Le BJP en parti de gouvernement?

6 Le BJP a toujours convoité la respectabilité des partis de gouvernement. Il s'est ainsi efforcé d'apparaître comme faisant partie intégrante du jeu politique légitime. Ce souci reflétait sans doute des préoccupations tactiques - la nécessité de trouver des alliés contre le Congrès, qu'exacerbait encore le mode de scrutin à un tour - mais aussi la prégnance du légalisme qui subsiste - certes de plus en plus difficilement - au coeur du système politique indien. L'opinion publique, notamment celle de la classe moyenne supérieure que façonne et exprime la presse de langue anglaise, attache au droit une importance qu'aucun parti ne peut tout à fait négliger. Si le noyau de l'électorat nationaliste hindou se compose de la petite classe moyenne (surtout des commerçants sans grande instruction) des villes de l'Inde hindiphone, le BJP avait précisément réussi à rallier ces dernières années des représentants de la classe moyenne supérieure, voire de l'élite (comme en témoigne la présence de retraités de la haute fonction publique et de l'armée parmi ses candidats aux élections de 1991). Ces milieux appréciaient en lui une force nationaliste, disciplinée et intègre plus qu'un défenseur de l'hindouisme, 
comme l'attestent les commentaires des quelques journalistes réputés ayant aussi rejoint la mouvance nationaliste hindoue ${ }^{4}$.

7 Les gouvernements contrôlés par le BJP à partir de 1990 se sont d'emblée employés à nuancer l'image acquise par leur parti d'une formation à programme unique (la construction du temple) et aux allures religieuses. Le gouvernement du Rajasthan a lancé un plan d'aide aux plus pauvres ; celui du Madhya Pradesh a entrepris d'alléger le fardeau des dettes supporté par les paysans et celui d'Uttar Pradesh s'est attaqué à la mafia locale, aux tricheries organisées par les étudiants lors des examens, aux constructions illégales qui se multiplient sur les terrains publics à mesure qu'augmente le prix du mètre carré dans les villes ${ }^{5}$. Ces mesures ont été bien reçues par la population. Le BJP a aussi inscrit à son crédit une diminution des émeutes entre hindous et musulmans dont la nébuleuse du RSS était un des principaux instigateurs lorsqu'elle était dans l'opposition; ceci a tendu à poser le BJP comme un parti apte à faire respecter la loi et l'ordre.

8 La popularité acquise sur un tel registre rapprochait le BJP du statut tant convoité de parti de gouvernement en dépit de mesures discriminatoires envers les minorités (à Bhopal, la capitale du Madhya Pradesh, les musulmans furent les premières victimes de l'élimination des constructions illégales). Si cette formation n'échappait pas à l'usure du pouvoir, notamment en raison de la corruption rapide de ses chefs locaux qui n'avaient guère goûté au pouvoir jusqu'alors; cette érosion était lente, comme en témoignent les succès du BJP lors des élections partielles de l'automne 1991 au Madhya Pradesh et en Uttar Pradesh. La principale difficulté finalement rencontrée par le parti tenait à l'attitude de ses militants et des autres composantes de la nébuleuse du RSS.

9 Les élus du BJP, dans les quatre Etats qu'il gouvernait, l'avaient emporté grâce à leur manipulation de thèmes nationalistes hindous dans le cadre de la campagne pour le temple de Ram. Il s'agissait, pour la plupart d'entre eux, de militants fortement idéologisés dont la candidature avait été retenue en raison de ce profil. Ces élus avaient acquis au fil des ans une "culture d'opposition" qui, parfois combinée à l'ascétisme hérité de leur passage au RSS, les portait à critiquer leurs propres gouvernements ; d'autant que les membres de ces derniers - accaparés par leur tâche et/ou rendus hautains par leurs nouvelles charges - se coupaient de leur base militante ${ }^{6}$. L'insatisfaction ressentie par cette dernière alimenta le factionnalisme dans un parti jusqu'alors réputé pour sa discipline - un autre trait hérité du RSS. Au cours de l'été et de l'automne 1992, des députés du Madhya Pradesh et de l'Uttar Pradesh organisèrent des pétitions visant le remplacement des chefs du gouvernement de ces deux Etats ${ }^{7}$.

Nul doute que l'inflexion nationaliste hindoue des chefs du BJP, qui contredisait leurs aspirations au statut de parti de gouvernement, refléta la nécessité de retrouver un terrain d'action commun à tout le parti. En 1992, l'introduction dans les écoles de ces Etats de manuels d'histoire où l'on vantait les gloires passées de la communauté majoritaire (les aryens y étant par exemple décrits comme le peuple originel de l'Inde) devait aider les gouvernants à réaffirmer un ancrage idéologique que leur quête de respectabilité semblait diluer.

Un parti sous pression

11 Les principales pressions en faveur d'une revalorisation par le BJP du registre hindou militant provenaient cependant de la VHP. Cette organisation présentait une structure dualiste puisque son "exécutif" se composait surtout d'hommes du RSS (comme Ashok Singhal) tandis que les religieux (chefs de sectes ou simples renonçants -sadhu -) 
dominaient le Margdarshak Mandal (le Cercle de ceux qui montrent la voie) et la Dharma Sansad (Assemblée du dharma) deux instances "délibératives" d'où l'organisation tirait l'essentiel de son autorité en raison du prestige dont jouissent encore les religieux dans la population hindoue. En dépit de cette configuration bicéphale, la VHP se caractérisa longtemps par une grande unité : la construction du temple restait son article de foi prioritaire, voire unique, qu'il s'agisse d'un symbole de la renaissance nationale hindoue (pour ses chefs issus du RSS) ou, pour les sadhu, d'une oeuvre plus religieuse, rendant son lustre à l'hindouisme et à ses représentants patentés dans une Inde laïque où ces derniers n'étaient plus guère reconnus.

La VHP, une fois posée la première pierre du temple en novembre 1989, décida, en février 1990, de laisser quatre mois au gouvernement Janata Dal de V.P. Singh qui venait de battre le Congrès (I) de Rajiv Gandhi, avec l'aide du BJP, pour faire admettre aux organisations musulmanes que la mosquée devait être déplacée. Le BJP, qui était partie prenante de la coalition au pouvoir et qui s'inquiétait du regain d'activité des séparatistes cachemiris, n'était pas étranger à cette concession de la VHP : il lui fallait apparaître responsable dans un contexte dominé par des enjeux extérieurs importants pour la nation. Après l'expiration du délai auquel elle avait consenti, la VHP annonça en juin 1990 qu'elle commencerait la construction du temple (baptisé Kar Seva - litt. "service action") le 30 octobre 1990.

13 Le BJP avait alors tout à gagner à s'associer au mouvement, voire à en prendre le contrôle: V.P. Singh refusait de mener sa politique de répression au Cachemire et risquait de diviser les hindous par sa promesse, le 15 août 1990, de réserver $27 \%$ des postes de l'administration centrale aux castes dites "arriérées" dont il espérait sans doute constituer sa base électorale et que convoitait aussi le BJP. Le président du parti, Advani, parcourut alors $10000 \mathrm{~km}$ pour défendre la cause de Ram à grand renfort de discours nationalistes hindous. Son périple devait le conduire à Ayodhya pour inaugurer la construction du temple le 30 octobre, mais V.P. Singh le fit arrêter peu avant. En dépit des forces de l'ordre déployées par le gouvernement du Janata Dal d'Uttar Pradesh, des milliers de kar sevak venus de toute l'Inde pour répondre à l'appel de la VHP, atteignirent Ayodhya le jour dit et prirent d'assaut la mosquée dont ils endommagèrent les murs et les dômes.

Les kar sevak, s'ils comprenaient quelques paysans et des sadhu, se recrutaient surtout parmi une jeunesse au profil sociologique hétérogène : le Bajrang Dal, les "jeunesses" de la VHP, comporte des étudiants de hautes castes, mais aussi des fils de paysans sans éducation, exaltés par la cause de Ram. Cette composition sociale explique en partie les tendances du Bajrang Dal à la violence et son manque de discipline qui contraste avec le RSS. Cette jeunesse constitue cependant une masse de manoeuvre idéale dans le cadre de la "stratégie instrumentaliste" en raison de sa promptitude à se mobiliser dans la rue. La répression de la Kar Seva fit quelques dizaines de morts, tous issus de cette jeunesse. Ces victimes furent aussitôt érigées en martyrs et contribuèrent ainsi grandement aux succès électoraux du BJP en 1991, en Uttar Pradesh tout du moins.

15 Jusqu'à cette date, le parti avait continué à bénéficier à plein de son association avec la VHP dans le cadre de la campagne pour le temple de Ram. La "stratégie instrumentaliste" lui avait en effet permis de manipuler ce symbole, avec la caution des religieux et d'en tirer un profit politique croissant. Dès lors que ces dividendes se traduisirent par l'installation d'un gouvernement du BJP en Uttar Pradesh en juin 1991, la gestion de cette association avec la VHP devint beaucoup plus délicate. Celle-ci, qui 
rappelait volontiers au chef du gouvernement de l'Etat qu'elle était à l'origine de son succès, exigeait que le nouveau pouvoir honore le mandat dont l'avaient chargé les électeurs : construire le temple. Kalyan Singh, porté à la tête du gouvernement de l'Etat, soucieux d'accréditer le statut de parti de gouvernement revendiqué par le BJP, s'efforçait de temporiser. En octobre 1991, il organisa à Ayodhya une commémoration des victimes de la Kar Seva afin, simplement, de donner des gages de son ancrage nationaliste hindou à des militants impatients - surtout au sein de la VHP. Cette célébration symbolique dégénéra cependant lorsque les militants en question attaquèrent la mosquée, endommagée pour la seconde fois. Le BJP ne pouvait reporter indéfiniment des mesures plus concrètes pour satisfaire cette base.

La difficile gestion du dilemme

16 La difficulté consistait pour le BJP d'Uttar Pradesh dans la nécessité de concilier son statut de parti de gouvernement et les demandes de militants auxquels il devait le succès de sa "stratégie instrumentaliste". A l'automne 1991, sous couvert de développer un complexe touristique, Kalyan Singh acquit 2,77 acres, dont l'essentiel avait déjà été acheté par la VHP, situés devant la mosquée et comprenant le site où avait été posée la première pierre. Telle que celle-ci se présentait, étant donné les plans du temple, la mosquée était condamnée par cette construction. Mais l'idée du BJP était de commencer à édifier le temple sur le terrain acquis par le gouvernement, de manière à fournir un premier motif de satisfaction et d'action aux militants ; pendant ce temps, évalué à deux années, la recherche d'un compromis avec les musulmans, dans le cadre légal, se poursuivrait. Dans cette perspective, les travaux commencèrent autour de la première pierre en juillet 1992.

La légalité de l'acquisition des 2,77 acres par le gouvernement avait toutefois été contestée par des organisations musulmanes qui arguaient du fait qu'un cimetière islamique composait l'une des parcelles. Le tribunal de l'Etat, saisi du dossier, demanda donc l'arrêt des travaux jusqu'à l'annonce de sa décision. La Cour Suprême devant laquelle il avait été fait appel réitéra cette demande. Les militants de la VHP et les sadhu assemblés à Ayodhya ne désarmant pas, Narasimha Rao, Premier ministre de l'Inde depuis juin 1991, négocia un compromis : la VHP fut autorisée à construire un autre temple dédié à Laxman, le frère de Ram dans la mythologie hindoue, à l'extérieur de la parcelle faisant l'objet du litige, et, surtout, le gouvernement central obtint quatre mois pour réunir une nouvelle fois les organisations représentatives des musulmans et la VHP afin de trouver un terrain d'entente.

Ce délai expira le 27 octobre sans que ces négociations n'aboutissent. La VHP annonça alors la reprise de la Kar Seva pour le 6 décembre. Au cours de l'automne, elle avait organisé une campagne de mobilisation intitulée Ram Paduka Yatra; il s'agissait de porter en procession, dans tous les cantons de l'Inde, 11500 paires de sandales (paduka) imitant celles que Ram, dans le Ramayana, avait abandonnées lors de son exil de 14 années hors d'Ayodhya - sous la pression d'une des femmes de son père, le roi, décidée à faire couronner son fils, Bharat, - et que ce demi-frère avait installées sur le trône dans l'attente du retour du véritable prince héritier ${ }^{8}$. Pour la première fois, cette agitation "instrumentaliste" n'avait pas rencontré l'écho escompté. Une certaine lassitude semblait s'installer dans la population en raison du scepticisme grandissant, après plusieurs tentatives avortées, quant à la capacité de la VHP de construire le temple. La désaffection dont elle commençait à souffrir conforta sans doute 
l'organisation dans sa détermination. Le jusqu'au-boutisme des sadhu s'affirmait tout particulièrement.

19 Le BJP était pris au piège de sa "stratégie instrumentaliste" : s'il se dissociait de la VHP, il se coupait d'un des principaux artisans de son récent essor électoral ; s'il endossait son militantisme, il ruinait son crédit de parti de gouvernement et, en cas de débordement de violence, il pouvait justifier une destitution du gouvernement d'Uttar Pradesh par New Delhi aux termes de la procédure dite "President's rule" ${ }^{\text {" }}$. Il chercha à résoudre son dilemme en essayant de faire renoncer la VHP à l'idée de la Kar Seva tout en prenant le relais de cette organisation dans la rue pour garder son image de défenseur de l'hindouisme.

20 A fortiori après que la Cour Suprême eut réitéré le 27 novembre 1992 qu'aucune construction ne pouvait avoir lieu sur les 2,77 acres en litige tant que le Tribunal d'Uttar Pradesh n'avait pas rendu son jugement, le BJP, soucieux de ne pas être accusé de transgresser la loi, milita auprès de la VHP pour que la Kar Seva ne soit qu'une cérémonie symbolique. Celle-ci aurait alors permis de remobiliser les militants sans tirer à conséquence. Ce compromis fut difficilement atteint dans les derniers jours de novembre. Le 29 novembre 1992, Advani pouvait déclarer, avec un soulagement non dissimulé : "la crise est derrière nous"10.

21 Aussitôt après, précisément pour réconforter la base nationaliste hindoue et consolider son image de défenseur de Ram, le BJP tendit à prendre la tête de la campagne en faveur de la Kar Seva: Advani et M.M. Joshi, le président du parti depuis février 1991, conduisirent à la fin novembre des processions qui traversèrent 28 districts avant de converger vers Ayodhya.

22 La contradiction entre la poursuite de cette "stratégie instrumentaliste" et l'effort simultané pour trouver un compromis qui ne remette pas en cause la respectabilité dont le BJP cherchait à se parer éclata le 6 décembre.

Depuis déjà plusieurs semaines, des kar sevak de l'Inde entière convergeaient vers Ayodhya. Cette fois encore il s'agissait surtout de jeunes hindous d'origine urbaine, mais on trouvait aussi des femmes, des paysans et, bien sûr, des sadhu. Ces personnes avaient été drainées par les appels de la VHP pour construire le temple. Bien des sadhu, en particulier, ne voulaient pas renoncer à cet objectif ${ }^{11}$. Or bien des jeunes kar sevak les considéraient comme leurs véritables leaders et n'étaient venus que pour eux, les chefs nationalistes hindous apparaissant trop calculateurs ${ }^{12}$. C'est d'ailleurs pour contrer cette influence que le RSS aurait choisi d'envoyer Advani et Joshi en tournée dans 28 districts d'Uttar Pradesh ${ }^{13}$. Mais cette surenchère avait encore plus échauffé les esprits et introduit la confusion. A Ayodhya même, des députés du BJP, réputés pour leur morgue oratoire, comme Uma Bharti, une renonçante, appelait les kar sevak à saisir l'occasion qu'offrait la Kar Seva pour sortir de la médiocrité ${ }^{14}$.

Le programme de la "Kar Seva symbolique" devait notamment consister, le 6 décembre à partir de $12 \mathrm{~h} \mathrm{15}$, à ramener, en procession, du sable et de l'eau de la Saryu (la rivière baignant Ayodhya) pour continuer le temple de Laxman et purifier, par des projections d'eau, les 2,77 acres acquis par le gouvernement et depuis "profanés par les politiciens"15. L'ensemble de l'opération devait être supervisé par des militants du RSS. Ceux-ci étaient notamment chargés d'encadrer les Kar sevak et d'assurer la protection des leaders du BJP et de la VHP qui devaient tenir un meeting au même moment. 

000 kar sevak étaient rassemblés suscita le premier mouvement de foule. A partir de 10 h 45, des militants du Bajrang Dal et de la Shiv Sena entreprirent de rompre le cordon des volontaires du RSS qui ceinturait la zone interdite aux travaux. Fondée à Bombay dans les années 1960, la Shiv Sena a d'abord eu pour vocation la défense des "fils du sol", maharashtriens, contre l'immigration des Indiens du sud accusés de les priver d'emplois. Au cours des années 1980, la Shiv Sena a élargi son nationalisme maharashtrien à un nationalisme hindou qui l'a rapprochée du BJP avec lequel elle a conclu des accords électoraux. Tout comme le Bajrang Dal, la Shiv Sena tend à recruter ses membres dans le milieu des jeunes hindous de castes intermédiaires et bloqués dans leur ascension sociale, qu'ils soient chômeurs désoeuvrés ou salariés aspirant à une vie plus gratifiante ${ }^{16}$.

Après que le cordon du RSS eut cédé à la pression des jeunes militants du Bajrang Dal et de la Shiv Sena, ce groupe parvint à prendre d'assaut la mosquée. Les statuettes de Ram et Sita en furent extraites avec soin puis les assaillants s'attaquèrent au mur extérieur en dépit des exhortations au calme prononcées par les leaders de la VHP, du BJP et du RSS. Ceux-ci se turent ensuite pendant deux heures au cours desquelles les trois dômes furent successivement détruits par de jeunes hindous que des sadhu aidaient en dégageant les branches surplombant la mosquée.

Ce mutisme a été interprété, notamment par le gouverneur de l'Uttar Pradesh comme l'indice d'un plan bien conçu à l'avance. Les chefs nationalistes hindous auraient fait en sorte de donner l'impression qu'ils étaient débordés par leurs troupes pour ne pas avoir à assumer la responsabilité d'une destruction qu'ils appelaient de leurs voeux ${ }^{17}$. Cette interprétation fut accréditée par le fait que les chefs en question ne redonnèrent de la voix que pour appeler les militants à bloquer les voies d'accès au site, de manière à retarder l'arrivée des forces de l'ordre, puis pour encourager les kar sevak en train de s'attaquer au dôme principal ${ }^{18}$. Certains commentateurs invoquent, à l'appui de cette thèse, la manière experte et les instruments, tout à fait adaptés, avec lesquels fut accomplie la destruction ${ }^{19}$.

Il est certain que bien des militants nationalistes hindous se félicitèrent de cet événement. Des cadres de rang intermédiaires ne s'en cachèrent pas dès le lendemain ${ }^{20}$. Pour ce qui est des leaders nationaux, cependant, il semble que d'une part certains de ceux qui étaient sur place se soient ralliés à l'idée d'une destruction après qu'elle eut commencé et que, d'autre part, nombre d'entre eux étaient fort embarrassés par ce "dérapage", voire désapprobateurs.

Kalyan Singh adressa sa démission au gouverneur de l'Uttar Pradesh six heures après le début de la destruction de la mosquée ${ }^{21}$. Advani démissionna peu après de son siège de leader de l'opposition à la Lok Sabha parce qu'il considérait "les démolitions comme regrettables. N'ayant pas réussi à les empêcher, je porte la responsabilité morale des événements"22. Le numéro deux du BJP après Advani, en termes de prestige et d'autorité morale, A.B. Vajpayee, depuis longtemps à la tête des modérés du parti et critique envers les excès de la "stratégie instrumentaliste", suggéra que le parti exprime ses regrets, par exemple au moyen d'un jeûne symbolique et déclara que la destruction de la mosquée compromettait tout projet de construction d'un temple ${ }^{23}$. Le chef du RSS, Balasaheb Deoras qualifia, lui, la destruction de "regrettable et inadéquate" et l'attribua à des "éléments" extérieurs ${ }^{24}$. S.S. Bhandari, un des viceprésidents du BJP, n'hésita pas à évoquer l'action d'"agents provocateurs" 25 . 
Si l'on admet que ces hommes ont été véritablement surpris par une destruction qu'ils ne voulaient pas voir s'accomplir dans ces conditions, ils ont alors été victimes de leur "stratégie instrumentaliste". Habitués à la discipline du RSS et confiants dans la capacité de ce mouvement à encadrer les militants ralliés à la cause au cours de l'agitation, ils n'ont pas perçu qu'un des pièges de cette stratégie consistait précisément à mobiliser des éléments que seule motivait l'adhésion aveugle, empreinte de religiosité et d'aspiration à l'"héroïsme", à la Cause de Ram, indépendamment de considérations plus idéologiques qui les auraient prédisposés à suivre les consignes d'organisations nationalistes hindoues.

Le piège s'est surtout refermé sur le BJP qui s'efforçait de temporiser pour ne pas nuire à sa respectabilité, mais aussi pour être en mesure de continuer à exploiter un enjeu populaire. Le dérapage s'est traduit pour lui par quatre revers :

a) la fiabilité de la masse de manoeuvre constituée par la jeunesse proche du Bajrang Dal et surtout par les religieux, si précieuse lors des opérations de mobilisation apparut affaiblie: ces deux ressources se révélaient d'un maniement délicat impliquant un effort de plus ou moins longue haleine pour discipliner ces troupes sur le modèle du RSS.

b) la classe moyenne supérieure, généralement attachée à la légalité républicaine est susceptible de prendre ses distances par rapport aux nationalistes hindous. Une recrue récente appartenant à ce milieu, le général Jacob - un militaire à la retraite qui fut un des héros de la guerre du Bangladesh - se montra perturbé par la démolition de la mosquée, tout comme les quelques représentants des milieux d'affaires présents au BJP (S.K. Modi ou J.K. Jain) ${ }^{26}$.

c) le parti a dû abandonner le pouvoir en Uttar Pradesh 18 mois après l'avoir conquis.

d) l'état-major du BJP est sorti divisé de cette crise car à côté des regrets - dont la sincérité est naturellement sujette à caution - qu'ont exprimé certains leaders, d'autres (comme Malkani ${ }^{27}$ ) ont reporté la faute sur la lenteur du tribunal d'Uttar Pradesh qui avait annoncé sa décision à propos des 2,77 acres pour le 11 décembre seulement. Inévitablement, la stratégie du parti était appelée, le 7 décembre au matin, à faire l'objet d'un débat au cours duquel des divisions anciennes entre "modérés" et partisans de la "stratégie instrumentaliste" seraient avivées.

Ces effets n'ont pas eu le temps de se développer parce que le pouvoir central, étant donné l'impact intérieur et international de la destruction du 6 décembre, a opté pour la répression des nationalistes hindous qui ont aussitôt resserré les rangs.

Violences internes, impact externe et répression.

Une nouvelle vague d'émeutes.

Dans les jours qui suivirent la destruction de la mosquée, l'Inde fut en proie à une vague d'émeutes entre hindous et musulmans qui fit officiellement plus de 1000 morts. Le 9 décembre, on dénombrait déjà 130 tués au Maharashtra, dont 94 à Bombay, 117 au Goudjerat (dont une majorité à Ahmedabad et Surat), 59 en Uttar Pradesh (où 26 districts seront finalement placés sous le couvre-feu), 48 au Madhya Pradesh (dont 34 à Bhopal), 28 en Assam, 16 en Andhra Pradesh (dont 6 à Hyderabad), 8 à Calcutta. Trois jours plus tard, le bilan par Etats s'établissait comme suit: Maharashtra, 256; Goudjerat, 253 ; Uttar Pradesh, 152 ; Madhya Pradesh, 94 ; Andhra Pradesh, 90 ; Assam, 75 ; Karnataka, 56 ; Rajasthan, 46 ; Bengale occidental, 37 ; Bihar, 22 ; Kerala, 15 ; Orissa, 3 ; Delhi, 3 ; Tamil Nadu, $2^{28}$. 

des années 1980 : des processions de militants hindous en forme de démonstrations de force scandaient des cris de victoire hindous ou des slogans injurieux pour les musulmans lors de la traversée de quartiers habités par ces derniers et la riposte dont ceux-ci étaient capables provoquait l'émeute ${ }^{29}$. Ce scénario se déroula avant l'attaque de la mosquée à Lucknow lorsque des militants de la VHP défilèrent dans un quartier musulman en entonnant des slogans provocants. L'émeute qui s'ensuivit ne fit cependant pas de victimes ${ }^{30}$.

41 Après la destruction, les violences opposèrent souvent les musulmans à la police. A Bombay, où l'on enregistra le plus grand nombre de morts, les musulmans, qui étaient descendus dans la rue pour manifester le lendemain de la destruction, furent la cible de la police qui n'était pas parvenue à les disperser ${ }^{31}$. Ce genre de pratiques semble avoir été courant, de sorte que nombre de victimes seraient en fait tombées sous les balles de la police. le rôle des provocations hindoues (souvent en formes de processions) pour déclencher l'émeute; la responsabilité croissante de la police, au moment d'expliquer le nombre des victimes (souvent musulmanes), qui est à rapprocher de son biais hindou de plus en plus marqué ; l'extension de la zone sujette à des violences intercommunautaires : si les régions hindiphones, traditionnellement les plus affectées par ce type de violences, présentent un bilan assez lourd, tous les Etats, y compris au sud, à l'exception du Tamil Nadu encore préservé, sont désormais touchés ; enfin, en corollaire de ce qui précède, la courbe des victimes d'émeutes reste ascendante : d'une moyenne de 400 tués par an au milieu des années 1980, on est passé à une moyenne annuelle au moins deux fois supérieure depuis les violences de 1989.

Une autre tendance qui s'est trouvée renforcée par la crise de décembre 1992 concerne l'interaction croissante des relations intercommunautaires avec l'environnement international puisque des violences ont aussi eu lieu, en réaction à la destruction de la mosquée, dans d'autres pays.

L'interaction avec le contexte international

e de cette interaction est depuis longtemps déterminant puisqu'une organisation comme le RSS est née en 1925, pour une bonne part, en réaction à la mobilisation des musulmans de l'Inde pour la défense du Califat d'Istanbul. A fortiori, depuis la formation du Pakistan, l'allégeance transnationale de la minorité musulmane alimente chez les nationalistes hindous l'idée selon laquelle cette dernière forme une cinquième colonne adossée à un chapelet d'Etats islamiques susceptibles d'encercler l'Inde.

En réaction à la destruction de la mosquée d'Ayodhya, le Pakistan ne s'est pas contenté d'une protestation officielle. Il a aussi interpellé l'Organisation de la Conférence des Etats Islamiques qui qualifia cet événement de "honteux"32. Cet effort pour internationaliser l'enjeu semblait intervenir dans une conjoncture propice puisqu'on observait à ce moment des réactions violentes non seulement au Bangladesh où des magasins et des temples hindous furent saccagés, mais aussi à Dubaï où un temple hindou fut attaqué ${ }^{33}$.

Il semble que dans ces derniers cas les exactions aient été le fait d'immigrés pakistanais et bangladeshis, les populations arabes du Moyen-Orient ne s'étant guère mobilisées faute parfois d'avoir la liberté de manifester leur solidarité musulmane dans des pays 
dont les régimes redoutent la montée en puissance de l'islamisme ${ }^{34}$. Les protestations populaires et/ou officielles qu'on observe dans les pays du Golfe persique ne pouvaient de toute façon qu'inciter le gouvernement indien à ramener l'ordre chez lui, étant donné qu'ils abritent des milliers de travailleurs d'origine indienne. Le regard des pays étrangers en général semble avoir été un facteur important dans la décision de mener une répression d'envergure. Dès la fin novembre, lorsque la crise était en gestation, Narasimha Rao avait d'ailleurs attiré l'attention de ses collègues du Congrès (I), moins portés à resituer l'enjeu dans son environnement international, sur les implications extérieures de l'affaire d'Ayodhya ${ }^{35}$. La dextérité avec laquelle les ambassades ont été informées de la ligne adoptée par le gouvernement indien et la façon dont elles ont distribué les communiqués et interviews où Narasimha Rao réaffirmait la vocation séculariste de l'Inde ${ }^{36}$ suggèrent une prise de conscience du poids du contexte mondial plus affirmée que par le passé.

Cette évolution peut s'expliquer par la dépendance croissante du pays envers le FMI, dont une délégation a d'ailleurs dû repousser sa mission d'expertise à cause des événements. l'Inde subit là une contrainte externe qui l'oblige à donner des gages de sa capacité à maintenir l'ordre chez elle. Par ailleurs, son gouvernement a sans doute vu quelques avantages diplomatiques à tenir le monde entier informé des événements en raison de l'ampleur des violences perpétrées au Pakistan contre la petite minorité hindoue (1,5 millions d'habitants, surtout concentrés au Sind). Dès la destruction de la mosquée, une trentaine de temples hindous furent pris d'assaut, les écoles restèrent fermées et les manifestations de rue se multiplièrent ${ }^{37}$. La demeure du Consul général de l'Inde à Karachi fit l'objet de violences au même moment. Le ministère indien des Affaires étrangères dénonça d'autant plus vigoureusement ces débordements qu'une semaine plus tard on aurait comptabilisé la destruction de 124 temples hindous sans que le gouvernement pakistanais ne semble mettre en oeuvre des moyens susceptibles de rétablir le calme. Le ministre adjoint, R.L. Bhatia, stigmatisa alors le "terrorisme d'Etat infligé aux communautés minoritaires" ${ }^{38}$ et souligna combien il contrastait avec l'effort indien pour mettre fin aux violences par la répression. L'Inde avait donc tout intérêt à prendre le monde à témoin.

Sa valorisation du contexte international s'inscrit sans doute aussi ici dans un projet tactique à visée interne : la focalisation sur l'antagonisme opposant l'Inde au Pakistan est un moyen traditionnellement utilisé, depuis le premier gouvernement d'Indira Gandhi surtout, pour ressouder les Indiens et mobiliser les hindous, dans un réflexe nationaliste, derrière le pouvoir. Il ne s'agit toutefois là que d'un aspect de la stratégie mise en oeuvre par Narasimha Rao et ses principaux ministres pour contrer les nationalistes hindous.

La contre-stratégie du pouvoir : légalisme et répression.

Dès sa nomination au poste de Premier ministre, la conduite de Narasimha Rao s'est caractérisée par un paradoxe: d'un côté, il se posait de façon plus crédible que ses prédécesseurs comme un héritier du sécularisme à la Nehru; d'un autre côté, il se refusait à toute confrontation avec les nationalistes hindous. Cette démarche, sans doute liée à l'absence d'une majorité solide au Parlement, se traduisit par une pondération destinée à gagner du temps sans compromettre l'essentiel. Dès sa prise de fonction, le Premier ministre fit par exemple voter une loi gelant le statut des lieux de culte, sauf celui d'Ayodhya, sur la base de leur situation en 1947. Parallèlement, il s'abstint de critiquer ouvertement le BJP dans l'espoir visible que celui-ci manifesterait 
la même retenue à son égard. Cette modération fut critiquée par Arjun Singh, un ministre du gouvernement central qui apparut bientôt comme le principal rival de Narasimha Rao dont il convoitait visiblement le poste.

Lorsqu'en juillet 1992, les nationalistes hindous se montrèrent à nouveau déterminés à construire le temple, le premier souci de Narasimha Rao fut de gagner du temps. Il obtint un répit de trois ou quatre mois qu'il chercha ensuite à mettre à profit pour enfoncer un coin dans le camp nationaliste hindou en dissociant certains religieux de la VHP. Pour la première fois, il décida de ne négocier qu'avec eux, les invitant même à son bureau de New Delhi où il leur aurait touché les pieds en signe de respect. Aucun chef religieux impliqué dans l'action de la VHP ne choisit cependant de rompre avec cette organisation, et Narasimha Rao dut envisager une autre parade à la pression nationaliste hindoue.

51 Lors du discours rituel que le Premier ministre prononce le 15 août, date anniversaire de l'indépendance, il exprima le souhait de voir édifier à Ayodhya un temple hindou à côté de la mosquée. Cette option était depuis longtemps privilégiée par la branche régionale du Congrès (I) en Uttar Pradesh étant donné la popularité acquise par le thème de la construction du temple ${ }^{39}$.

52 Cette tactique n'ayant pas porté les fruits escomptés, Narasimha Rao réunit pour la énième fois des représentants d'organisations musulmanes et de la VHP pour des pourparlers dont il ne sortit rien. L'enlisement de ces négociations amena Narasimha Rao à imaginer de confier au gouvernement la construction du temple évoquée dans son discours du 15 août $\mathrm{t}^{40}$, mais les réticences de certains leaders musulmans, soucieux de ne rien changer à Ayodhya, et surtout le refus farouche des nationalistes hindous qui ne voulaient pas voir capter le bénéfice de l'opération par le Congrès (I) semblent avoir dissuadé le Premier ministre de poursuivre dans cette voie.

53 Face à la détermination nationaliste hindoue de construire le temple, en novembre 1992, Narasimha Rao adopta une ligne plus ambitieuse consistant à isoler le BJP de façon méthodique. Le 19 novembre, il réunit le Congress Working Committee pour recevoir la caution de l'équipe dirigeante de son parti ${ }^{41}$. Il s'efforça ensuite, avec succès, d'amener les porte-parole musulmans les plus militants à davantage de modération de manière à ne pas légitimer, en retour, le ton agressif des nationalistes hindous ${ }^{42}$. Le 24 novembre, il présida une séance spéciale du National Integration Council - institution fondée en 1961 par Nehru pour résoudre les tensions intercommunautaires - que le BJP boycotta. Il reçut un mandat unanime de la part de tous les autres partis politiques, la résolution stipulant:

54 "La réunion du NIC, après avoir considéré tous les aspects du conflit autour de la Babri Masjid et de la Ramjanmabhoomi et le rapport du gouvernement, apporte son appui le plus complet [whole-hearted] et sa coopération à toutes les démarches que le Premier ministre juge essentielles pour faire respecter la Constitution, la loi et les décisions judiciaires" 43 .

55 Dans un troisième temps, précisément, la Cour Suprême réitéra le 28 novembre le caractère illégal que revêtirait toute opération de construction (ou de démolition) sur le site d'Ayodhya. Son arrêt précisait que les juges n'avaient aucune objection envers la "Kar Seva symbolique" pour laquelle avaient opté les nationalistes hindous.

56 Ceux-ci avaient finalement été placés par Narasimha Rao face à l'échiquier politique dans son ensemble et aux institutions les plus hautes de la République (la Cour 
Suprême, gardienne de la Constitution, mais aussi le Parlement où le Congrès, comme les autres partis d'opposition, ne ménageaient pas le BJP ${ }^{44}$ ). Le Premier ministre n'apparaissait ici que comme un arbitre en retrait qui se réservait le droit de sanctionner toute atteinte au cadre constitutionnel.

Au reste, il se contenta de mettre à la disposition de Kalyan Singh les troupes (80 compagnies de la Central Reserve Police Force) qu'il jugeait indispensable à la protection de la mosquée; il excluait d'y avoir recours lui-même comme aurait pourtant pu l'y autoriser l'article 355 de la Constitution aux termes duquel le pouvoir central a le devoir d'intervenir dans un Etat fédéré lorsque celui-ci est exposé à un péril extérieur ou menacé par des désordres intérieurs. D'une part, Narasimha Rao voulait éviter d'être à l'origine d'une répression des kar sevak comparable à celle menée en octobre 1990 par le Janata Dal qui s'était ainsi aliéné une partie de l'électorat hindou, notamment en Uttar Pradesh. D'autre part, il souhaitait laisser à Kalyan Singh la responsabilité d'une telle action ou de l'absence d'action : s'il donnait l'ordre de tirer, il serait mis en cause au sein même du camp nationaliste hindou; s'il s'abstenait d'intervenir et laissait se développer la violence, voire détruire la mosquée, il devrait assumer une incapacité à maintenir l'ordre, voire à faire respecter les décisions judiciaires, ce qui justifierait sa destitution. C'est ce qui se produisit puisqu'au moment où Kalyan Singh démissionnait, Narasimha Rao lançait la procédure visant à instaurer la "President's Rule" en Uttar Pradesh.

Le Premier ministre était donc parvenu à ses fins en se contentant de sanctionner les infractions au Droit commises par le BJP. Sa stratégie, d'un légalisme scrupuleux, avait cependant permis la destruction de la mosquée, ce qui suscita les critiques du Janata Dal -toujours prompt à s'ériger en défenseur des musulmans, celui-ci demanda la démission de Narasimha Rao - et de nombreux congressistes qui redoutaient notamment d'avoir perdu la confiance - et donc les voix - des musulmans choqués par l'événement. Arjun Singh, depuis longtemps partisan d'un durcissement du pouvoir à l'égard des nationalistes hindous, voyait sa position renforcée au sein du Congrès ${ }^{45}$.

Cette évolution du rapport des forces factionnelles au sein de cette formation explique sans doute en partie la promesse de Narasimha Rao de reconstruire la mosquée et l'intensification de la répression dans les jours suivant le 6 décembre. Le 8 décembre, Advani, Joshi, U. Bharti, Singhal et d'autres leaders de la VHP furent arrêtés en vertu des articles 153 et 505 du Code pénal (c'est-à-dire pour avoir perturbé "l'harmonie intercommunautaire" et prononcé des discours enflammés). Le 10 décembre, le RSS, la VHP, le Bajrang Dal et, pour faire bonne mesure, la Jamaat-e-Islami et l'Islamic Sevak Sangh, deux organisations à l'islam militant mais de modeste ampleur, furent interdits pour deux ans. Enfin, le 21 décembre, le pouvoir central instaura la "President's Rule" dans les trois Etats encore gouvernés par le BJP (Himachal Pradesh, Rajasthan et Madhya Pradesh). Au plan juridique, cette décision ne reposait sur aucune base solide, le BJP n'étant pas interdit et ces gouvernements, qui disposaient d'une solide majorité, n'ayant pas moins bien géré la vague de violence que ceux du Maharashtra ou du Goudjerat.

De façon générale, la pertinence stratégique de ce durcissement de la répression est pour le moins incertaine. Elle a sans doute été décidée sous la pression d'Arjun Singh, en tout cas en relation avec ses prises de position parce qu'il était susceptible de dénoncer la faiblesse de Narasimha Rao pour mieux apparaître comme la solution alternative au poste de Premier ministre. Cette spéculation, si elle se confirme, illustre 
l'ampleur des dommages que les luttes pour le pouvoir au sein du Congrès (I) continuent de causer à la vie publique. En l'occurrence, l'intensification de la répression risque d'être contre-productive.

61 Tout d'abord, elle a eu pour effet de ressouder le camp nationaliste hindou menacé par de nouvelles dissensions. Si au lendemain de la destruction de la mosquée, le BJP se trouvait partagé et même repentant pour certains de ses membres, en tout cas acculé à la défensive, notamment au Parlement ${ }^{46}$, le surlendemain, après l'arrestation de ses chefs, il s'était remobilisé, ses députés bloquant le fonctionnement de la Chambre basse par des slogans tels que "nous construirons le temple [à Ayodhya]" et "la mosquée ne sera pas reconstruite, le gouvernement ne peut faire cela"47. Dans le même temps, le RSS coordonnait la riposte du camp nationaliste hindou.

fut chargé d'organiser des meetings et des séminaires d'explication; la Sri Ram Kar Seva Samiti, un mouvement lié à la VHP et présidé par un religieux, se vit confier la tenue de réunions de propagande dans les temples; enfin, le RSS, en remplacement des sessions quotidiennes dans les shakha suspendues du fait de l'interdiction, prévît des réunions chez des swayamsevak chevronnés et "personnalités influentes" au plan local ${ }^{48}$. Il s'agissait sans doute là des dispositions les plus efficaces étant donné que le réseau du RSS comporte à présent 37000 branches, ce maillage de l'espace social surtout dense au nord -représentant 2,5 millions de swayamsevak. Le ministre de l'Intérieur, S.B. Chavan, ne dissimula d'ailleurs pas son scepticisme vis-à-vis de l'interdiction du RSS ${ }^{49}$ dont seulement 3000 militants ont été arrêtés, les principaux cadres étant passés à la clandestinité ou n'ayant pas été inquiétés. Or, le RSS compte à présent 7000 pracharak environ.

Le caractère contre-productif de la répression à l'encontre des nationalistes hindous ressort d'un sondage de MARG, un institut dont la fiabilité s'est trouvée confirmée lors des dernières élections ${ }^{50}$. Si 55,2 \% des personnes interrogées approuvent l'instauration de la President's rule en Uttar Pradesh, elles ne sont que 37,1 \% à penser de même pour les autres Etats.

64 Ces chiffres sont en outre à resituer dans un ensemble plus préoccupant encore : si 52,6 $\%$ des personnes interrogées désapprouvent la destruction de la mosquée, 54,2 \% de celles interrogées dans le Nord de l'Inde l'approuvent, et 65,8 \% de cette même catégorie désapprouvent le projet de la reconstruire émis par Narasinhao Rao. Celui-ci n'apparaît d'ailleurs comme le meilleur Premier ministre qu'à $17,9 \%$ des personnes interrogées dans le Nord de l'Inde (contre $21,2 \%$ pour Vajpayee et $10,1 \%$ pour Advani), le Sud lui restant acquis à $28 \%$ seulement. Le clivage de plus en plus marqué entre un Nord travaillé par la thématique communaliste et un Sud fidèle à un certain sécularisme identifié au Congrès (I) est défavorable à ce dernier en raison du poids démographique et politique de la zone hindiphone dont les cinq grands Etats représentent 230 sièges sur 543 à la Chambre basse. Ce sondage de MARG laisse d'ailleurs apparaître que le Congrès (I) n'y conserverait que 233 sièges, tandis que le BJP passerait de 119 à 170 sièges $^{51}$.

Le pouvoir central a d'ailleurs très vite atténué ses premières mesures de répression. Des leaders nationalistes hindous de premier rang tels qu'Advani ont été libérés sous caution et il est possible que le pouvoir essaiera de reporter leur procès par crainte d'une mobilisation hindoue. de construire dans l'année, de façon simultanée, un temple et une mosquée à Ayodhya. 
La difficulté réside cependant toujours dans le site à choisir pour ces deux bâtiments : les musulmans sont soucieux de voir réutilisé le terrain qu'occupait la Babri Masjid ; or, après avoir détruit celle-ci, les kar sevak ont réinstallé les idoles de Ram et Sita à l'endroit où elles avaient été placées dans la mosquée puis ont construit un temple sommaire autour. Revenant sur sa fermeté initiale, le pouvoir a accédé aux demandes des nationalistes hindous et de sadhu qui menaçaient de poursuivre un jeûne jusqu'à la mort quant au culte à organiser dans ce temple : les dévots pourront finalement venir y contempler les idoles, ce qui compliquera encore le règlement du problème.

La crise de décembre 1992 constitue sans doute un tournant dans la trajectoire politique de l'Inde. Sur un plan social, la coexistence, de moins en moins pacifique il est vrai, entre les communautés souffrira sans doute longtemps de la destruction de ce symbole que constituait la mosquée d'Ayodhya et des violences qui l'ont suivie. Hormis les musulmans, les autres minorités risquent de voir dans le fait que le pouvoir central a permis cette destruction, la confirmation d'un statut qu'elles considèrent subordonné.

Sur le plan politique - où nous nous sommes situés dans cette note - la voie pour sortir de cette crise parait étroite. Le BJP avait semblé affaibli immédiatement après la destruction de la mosquée parce qu'il s'était laissé prendre au piège de sa "stratégie instrumentaliste": les jeunes militants et les religieux qu'il avait jusqu'alors utilisés avec grand profit comme masse de manoeuvre et instruments de mobilisation sur un thème religieux porteur, par ce débordement de violence, ayant remis en cause ses prétentions au statut de parti de gouvernement, ranimé la critique de son courant modéré et indisposé les membres de la classe moyenne récemment attirés dans sa mouvance.

L'ampleur de ce revers a semble-t-il été plus qu'atténuée par la politique du gouvernement central. Narasimha Rao avait fort bien manoeuvré en isolant le BJP et en faisant assumer à Kalyan Singh des violences qui justifiaient sa destitution. Cette tactique fondée sur le respect du Droit avait toutefois été mise en oeuvre au prix de la destruction de la mosquée, ce qui aliénait une partie de l'électorat musulman et, audelà, donnait à penser que le pouvoir avait fait preuve de faiblesse, par excès de légalisme, dans sa défense du sécularisme.

Le durcissement de la répression qui s'ensuivit risque de compliquer le règlement de la crise à moyen terme. La prise de contrôle de l'Uttar Pradesh par New Delhi, légitime, lui aurait permis de construire un temple et de rebâtir la mosquée côte à côte tandis que le BJP continuait à s'user au pouvoir dans d'autres Etats. Sans doute s'agissait-il là du plan initial de Narasimha Rao. La multiplication des arrestations, l'interdiction du RSS et la destitution, difficile à justifier, de trois autres gouvernements du BJP, sans doute sous la pression d'Arjun Singh, ont remobilisé les nationalistes hindous et risquent de les faire apparaître comme les victimes d'une cause sacrée pour les hindous. De là les efforts du pouvoir pour atténuer certains aspects d'une répression assez impopulaire, dans le Nord du pays surtout. Ces atermoiements, liés au jeu des factions, peuvent cependant donner du gouvernement l'image d'un pouvoir inconstant au moment où il est engagé dans une épreuve de force de longue haleine : l'impression de flottement ainsi créée peut détourner du Congrès (I) certains électeurs du Nord et conforter les nationalistes hindous dans leur détermination à obtenir des élections anticipées au Parlement.

71 Un premier indice du succès ou de l'échec du pouvoir central dans son effort pour contrer les nationalistes hindous sera fourni par les élections dans les Etats dont les 
gouvernements ont été destitués. Un retour en force du BJP constituerait un revers très sérieux pour le Premier ministre et le sécularisme indien. D'ici là, Narasimha Rao devra cependant surmonter l'épreuve de la session budgétaire de février-mars où, faute d'une majorité absolue, il lui faudra obtenir la neutralité bienveillante de députés de gauche ou du centre gauche pour une loi de finances rendue très stricte par les conditions de l'ajustement structurel.

\section{NOTES}

1. Cet article s'inscrit dans le prolongement de celui publié dans le numéro 5 (printemps 1992) de Cultures et Conflits sous le titre "Les émeutes entre hindous et musulmans : essai de hiérarchisation des facteurs culturels, économiques et politiques". 2. Pour plus de détails sur les circonstances de cette remise à l'honneur de l'enjeu d'Ayodhya, voir la quatrième partie de Jaffrelot, (C.) Les nationalistes hindous Idéologie, implantation et mobilisation des années 1920 aux années 1990, Paris, Presses de la FNSP, 1993.

3. Le conditionnel est ici de rigueur car ces vestiges archéologiques peuvent aussi bien avoir appartenu à un temple shivaïte ou jain. Les motifs dont ils sont ornés semblent en tout cas exclure une origine vishnouite, le milieu sectaire dans lequel s'inscrit la figure de Ram. Le temple antique, s'il a existé, ne lui aurait donc pas été dédié (C.B. Asher attribue une origine shivaïte aux piliers présents dans la mosquée dans Architecture of Moghol India - The New Cambridge History of India I - III, Cambridge, Cambridge University Press, 1992).

4. Voir l'allocution d'Arun Shourie en tant que président d'honneur de la cérémonie annuelle du RSS à l'occasion de la fête de Dasahara (Organiser 18 octobre 1992, p. 9) et les éditoriaux de Swapan Das Gupta dans Sunday, tel que "Wanted : politics" (Sunday 22-28 novembre 1992, p.8)

5. Ibid., 6-12 décembre 1992, p. 92.

6. J'ai examiné ce phénomène dans la dernière section de "The BJP in Madhya Pradesh : networks, strategy and power" in Pandey, G. ed. Hindus and others : The question of identity in India today, New Delhi, Viking, 1993.

7. India Today, 15 novembre 1992, p. 17 et p. 31.

8. Cette explication est plus élaborée dans l'organe du RSS, Organiser, Deepawali Special, 1992, p. 11. Voir aussi India Today 31 octobre 1992, p. 37.

9. Le recours à la "President's Rule", par laquelle le pouvoir central prend en main les affaires d'un Etat dont le gouvernement est alors démis et l'assemblée dissoute, est justifié, d'après l'article 356 de la Constitution, dans les cas où les affaires publiques ne peuvent être administrées conformément à la Constitution.

10. The Times of India 29 novembre 1992.

11. The Times of India 5 décembre 1992.

12. Ibid. 5 décembre 1992.

13. Ibid. 6 décembre 1992 ; 
14. Pour comprendre l'impact d'un tel discours (par exemple sur un jeune kar sevak d'Andhra Pradesh, ibid. 5 décembre 1992), il faut songer à l'aspiration qui travaille toute une jeunesse indienne à devenir "un héros" sur le modèle de Ram, mais plus encore sur celui des acteurs de cinéma. Ram ressemble d'ailleurs à ces derniers sur les affiches nationalistes hindoues où il apparaît musculeux, armé d'un trident, bravant une mer qui, bien que grossie par la tempête, ne lui arrive pas aux genoux.

15. Ibid. 6 décembre 1992.

16. Voir Gérard Heuzé, "Les Shiv Sena(s). Des bureaux de chômage au nationalhindouisme ? Réflexions à partir d'une étude de cas au Madhya Pradesh en 1991", Annales, n 4-5, juil.-octobre 1992, p. 841-864.

17. The Times of India 7 décembre 1992.

18. The Pioneer 7 décembre 1992.

19. The Times of India 9 décembre 1992, p. 14.

20. The Times of India 9 décembre 1992, p. 14.

21. The Pioneer 7 décembre 1992, p. 1.

22. Cité dans The Times of India 9 décembre 1992, p. 1.

23. Ibid. 7 et 13 décembre 1992, p. 4 et p. 11.

24. Ibid. 8 décembre 1992, p. 4.

25. The Pioneer 7 décembre 1992, p. 5.

26. The Times of India 13 décembre 1992, p. 11.

27. The Pioneer 7 décembre 1992, p. 5.

28. The Pioneer 12 décembre 1992, p. 4.

29. Pour une présentation plus complète de ce "modèle", voir Jaffrelot, C. "Les émeutes entre hindous et musulmans", art. cit.

30. The Pioneer 28 novembre 1992.

31. The Pioneer 12 décembre 1992, p. 9.

32. The Pioneer 9 décembre 1992, p. 7 ; The Times of India 8 décembre 1992, p. 3.

33. De telles attaques furent aussi organisées dans trois villes d'Angleterre par des musulmans originaires du sous-continent indien (The Pioneer 9 décembre 1992, p. 7).

34. The Times of India 13 décembre 1992.

35. The Statesman 19 novembre 1992. Après la destruction de la mosquée, il déclarera devant le Congress Parliamentary Party : "le monde entier nous regarde et les membres du parti doivent être à la hauteur de la situation" (The Times of India 9 décembre 1992, p. 3).

36. Entre le 11 et le 30 décembre, l'Ambassade de France a distribué une dizaine de communiqués officiels représentant une quarantaine de pages.

37. Benazir Bhutto, principal leader de l'opposition, appela même à une grève générale (The Pioneer 9 décembre 1992, p. 7).

38. Communiqué du 16 décembre 1992 distribué par les services d'information de l'Ambassade de l'Inde à Paris.

39. Le 3 décembre 1992, un millier de congressistes défilèrent à Faizabad - le chef-lieu $\mathrm{du}$ district où se trouve Ayodhya - en scandant : "Ram, nous sommes venus construire le temple" et "Construire le temple et sauver la mosquée" (The Times of India 5 décembre 1992).

40. The Times of India 16 octobre 1992.

41. The Statesman 19 novembre 1992.

42. India Today 15 décembre 1992, pp. 28-29.

43. The Statesman 24 novembre 1992. 
44. Advani fut ainsi interpellé le premier jour de la session parlementaire d'hiver aussi bien par le ministre de l'Intérieur que par des intervenants communistes et du Janata Dal, ce qui confirmait l'unanimité du NIC (The Pioneer 25 novembre 1992).

45. Arjun Singh fut avec Narasimha Rao le principal orateur lors de la réunion du Congress Parliamentary Party le 9 décembre 1992 (The Pioneer 9 décembre 1992, p. 4). 46. A la Chambre basse, la séance dû être interrompue à $14 \mathrm{~h}$ en raison du désordre provoqué par les slogans hostiles au BJP auxquels les députés de ce parti - bien moins nombreux qu'à l'ordinaire - ne répondaient d'ailleurs pas (The Times of India 8 décembre 1992, p. 1).

47. Ibid. 9 décembre 1992, p. 3.

48. The Pioneer 11 décembre 1992, p. 5.

49. The Times of India 13 décembre 1992, p. 11.

50. Il est toutefois probable que ce sondage, comme les autres, sur-représente sensiblement l'opinion de la classe moyenne urbaine. En l'occurrence, cela ne le rend que plus intéressant.

51. India Today 15 janvier 1993, pp. 14-20.

\section{RÉSUMÉS}

La scène publique de l'Inde est dominée depuis la fin des années 1980 par un conflit entre Hindous et Musulmans dont l'objet est le site d'Ayodhya, lieu saint pour les Hindous qui souhaiteraient y édifier un temple à la place de la mosquée. Ce conflit ne saurait se résumer exclusivement, loin s'en faut, à un affrontement religieux, où le facteur "culturel" serait seul en mesure d'expliquer son existence. Toutefois les succès électoraux des mouvements nationalistes hindous, en 1990 et 1991, et notamment ceux du BJP, parti du peuple indien, s'expliquent en partie par leur "stratégie instrumentaliste" centrée autour du conflit et de l'enjeu d'Ayodhya. Cette stratégie qui impliquait que le BJP poursuive ses efforts de mobilisation est entrée en contradiction avec son aspiration à devenir un parti de gouvernement susceptible de conquérir le pouvoir central. La crise de décembre 1992, provoquée par la destruction de la mosquée, a révélé les limites de cette stratégie même si au demeurant la répression orchestrée par New Delhi les a transformé en victimes.

Since the late 1980s, India's public life is dominated by a Hindu-Muslim dispute concerning the site of Ayodhya, sacred to Hindus, who wish to build a temple in place of the existing mosque. This conflict is far from being an exclusively religious or cultural one. Nonetheless, the electoral success of Hindu nationalist movements in 1990 and 1991, particularly of the BJP (Indian People's Party), can be partly explained by their " instrumentalist " use of Ayodhya. This strategy implied that the BJP should continue mobilising the masses, although this was inconsistent with its desire to play a governmental role. The December 1992 crisis, at the time when the mosque was destroyed, revealed the weakness of this strategy, even though the repression set in motion by New Delhi turned the attackers into victims. 
INDEX

Index géographique : Inde

Mots-clés : émeutes, répression

Index chronologique : 1990 - 2000 